Jorge A. Manrique Guzmán' Beatriz del C. Chávez Reátegui ${ }^{2}$

'Docente de la Facultad de Odontología. Universidad Nacional Federico Villarreal.

2Docente del Departamento Académico de Clínica Estomatológica. Facultad de Estomatología. Universidad Peruana Cayetano Heredia.

\section{Correspondencia}

Jorge Manrique Guzmán

Calle Arq. Juan Benites 289 - 301 - Lima 41, Perú. Telefono 226374

e-mail: jorgemanguz@ hotmail.com

Recibido : 25 de junio de 2010

Aceptado : 12 de agosto de 2010

\title{
Adaptación del colado en prótesis fija y removible en pacientes de la clínica odontológica de la UNFV
}

Manrique-Guzmán JA, Chávez-Reátegui BC. Adaptación del colado en prótesis fija y removible en pacientes de la clínica odontológica de la UNFV. Rev Estomatol Herediana. 2010; 20(3):137141.

RESUMEN

El presente estudio, fue diseñado para identificar la frecuencia de adaptación de los colados en prótesis fija y removible en pacientes de la clínica odontológica de la Universidad Nacional Federico Villarreal, tomando como muestra 100 trabajos que ingresaron al laboratorio de la facultad, el $38 \%$ fueron prótesis parcial re movible de cromo cobalto y el $62 \%$ prótesis fija. De esta muestra, el 78\% de las prótesis parciales removibles, requirieron desgastes internos para su adaptación, ya sea de los ganchos, retenedores y/o opositores como de los apoyos, el 10\% se debió repetir y tan solo el $12 \%$ adaptó sin ningún tipo de desgate, de las prótesis fijas, en promedio, e1 $80 \%$ debió recibir: algún tipo de desgaste para su adaptación y un $18 \%$ debió de repetirse, mientras que tan solo un $2 \%$ no requirió de ningún tipo de desgaste. Estos resultados nos indican una alta frecuencia de errores y fallas en los colados que repercuten directamente en la eficiencia y calidad del servicio.

Palabras clave: DENTADURA PARCIAL FIJA / DENTADURA PARCIAL REMOVIBLE / OCLUSIÓN DENTAL.

Adjustment of the cast in fixed and removable prosthesis in patients of the dental clinic of National University Federico Villarreal

ABSTRACT

This study was designed to identify the frequency of adjustment of the cast in fixed and removable prosthesis in patients in the dental clinic of National University Federico Villarreal, taking a sample of 100 models that were sent to the laboratory of the dental school, 38\% were removable partial dentures cobalt chromium and $62 \%$ fixed prosthesis. From this sample, $78 \%$ of the removable partial dentures, required trimming for their adaptation, either of the retaining the hooks and / or opponents of support, $10 \%$ was due to repeat and only $12 \%$ adjusted without any wear rate of fixed prostheses, on average, $80 \%$ had received some type of trimming for adaptation and $18 \%$ had to be repeated, while only $2 \%$ did not require any kind of trimming. These results indicate a high frequency of errors and mistakes in the cast that directly affect the efficiency and quality of service.

Key words: FIXED PARTIAL DENTURE / REMOVABLE PARTIAL DENTURE / DENTAL OCCLUSION.

\section{Introducción}

Es de conocimiento de todos los profesionales de la salud que siempre la calidad de la atención fue, es y será lo mas importante en un servicio sea medico u odontológico y en la actualidad se ha convertido en una necesidad para todo profesional de la salud conocer como se esta manejando este aspecto ya que son pocos los estudios que se refieren concretamente a la calidad de la atención y menos aún su evaluación el campo de la odontología $(1,2)$.

Gurnik y Twan (1995) (3) cita que instituciones encargadas por velar por la salud publica del planeta como la Organización Mundial de la Salud (OMS) ha definido a la calidad de un servicio como el alto nivel de excelencia profesional, uso adecuado y eficiente de los recursos disponibles, mínimo riesgo para el paciente, alto grado de satisfacción por parte del paciente y el impacto que pueda tener el servicio en la salud.

Los términos eficiencia y eficacia aún son motivo de estudio importante para analizar la calidad de la atención. Eficiencia, capacidad de reducir al mínimo los recursos usados para alcanzar los objetivos trazados. Eficacia, resultado en relación a las metas y el cumplimiento de los objetivos trazados. Es importante priorizar las tareas y realizar en orden de precedencia aquellas que contribuyen a alcanzar los objetivos y metas previstas, para poder asegurar y conseguir el fin trazado (2-4).
La evaluación constante de la calidad en el servicio es de suma importancia, es el medio por el cual el proceso es observado y perdiendo emitir: un juicio y poder identificar en que estamos errando o acertando y de esta manera poner los correctivos del caso que redundaran en una mejor atención (3).

En el campo de la estomatología, se realizan una serie de tratamientos clínicos que van desde los preventivos hasta los restaurativos, pasando por lo quirúrgicos y otro tipo de procedimientos. En el área de la restauración, se encuentran los tratamientos protésico, donde no solamente interviene el profesional odontólogo sino el personal auxiliar de laboratorio capacitado que es el que confecciona la aparatología protésica. 
Los tratamientos medicoodontológicos tienen riesgos que los profesionales los deben controlar y solo cuando se controlan estos riesgos y se maneja al paciente de una manera "adecuada", se puede hablar de éxito en el tratamiento; específicamente, hablando de tratamientos protésicos el éxito, es determinado a través de la longevidad de la prótesis, preservación de la salud sea pulpar y/o gingival de los dientes involucrados así como de los remanentes y finalmente por la satisfacción del paciente donde hoy en día, la estética se ha convertido en parte fundamental en la definición de "salud oral" (6-8).

El paciente espera y tiene la expectativa que el odontólogo satisfaga plenamente su exigencia en cuanto a lo funcional y lo estético; del mismo modo, el odontólogo espera que el técnico dental también satisfaga sus expectativas brindándole un trabajo protésico en el cual no tenga que hacer mayores correcciones o desgastes, un trabajo de calidad (9).

Las Facultades de Odontología de las diferentes Universidades, tienen asignaturas clínicas en su plan curricular. Estas asignaturas suelen ubicarse desde mediados de la carrera hasta el final, donde los estudiantes realizan trabajos clínicos de diferente índole entre ellos las prótesis, sean fijas y/o removibles.

Los estudiantes de la clínica integral del adulto de la Facultad de Odontología de la Universidad Nacional Federico Villarreal, deben de cumplir una serie de actividades clínicas entre las que figuran tratamientos protésicos los cuales son iniciados en el paciente en la clínica odontológica y elaborados en el laboratorio dental de la Facultad.

Evidentemente, como activad psicomotriz, está impregnada de un margen de error, que se presenta tanto en la clínica (con el paciente) como en el laboratorio (con los modelos de trabajo) y es muy importante la coordinación de ambos para entender y controlar estos posibles errores (10).

Modaffore et al. (2000) (11), en una revisión de la literatura, encontraron que muchos profesionales no ponen el suficiente cuidado al planificar, operar y orientar al laboratorio para trabajos de prótesis parcial removible y prótesis parcial fija. Refieren que muchas de las fallas y repeticiones ocurren por una no adecuada comunicación del odontólogo y el técnico de laboratorio responsable del trabajo.

Del mismo modo, Aquilino y Taylor (1984) (12), encontraron que los dentistas están delegando gran parte de la responsabilidad a los técnicos, en lo que respecta a trabajos de prótesis fija. Los técnicos investigados, estaban insatisfechos con la calidad del trabajo recibido; las quejas incluían insuficiente información sobre la autorización del trabajo, impresiones deficientes y registros inadecuados.

Las repeticiones de un trabajo, están ligadas directamente en la calidad del servicio, la repetición de los trabajos de laboratorio dental afectan la eficiencia del servicio dental, debido a la duplicidad de los gastos y la insatisfacción de los pacientes por el mayor tiempo utilizado para su tratamiento $(12,13)$.

Por otro lado, Delgado (1997) (14), menciona que la repetición de los tratamientos dentales; afectaría la calidad de los servicios odontológicos por la duplicidad en los gastos, al tener que utilizar materiales adicionales, y el mayor tiempo utilizado para el tratamiento del paciente, afectando la satisfacción de éste. En el mismo sentido Horowitz (1991) (15) y Pérez (1997) (16), mencionan que el excesivo número de citas afectan la calidad de los servicios de salud puesto que redundan en la no satisfacción de los pacientes, siendo este uno de los mayores problemas.

Cuando se tienen que hacer repeticiones de los trabajos de laboratorio en prótesis dental, se tiene que incrementar el numero de citas y al respecto, Ccahuana (1997) (17), realiza un estudio para determinar el número de citas empleadas por pacientes según el tipo de tratamiento en rehabilitación oral y endodoncia efectuado por los alumnos del curso de Clínica Integral del Adulto en la UPCH, encontrando que existen diferencias significativas entre el número ideal de citas en relación al número real en los tratamientos de prótesis y endodoncia, así como diferencias significativas entre el número de días empleado y el número de días ideal en los tratamientos de prótesis y endodoncia.

Por otro lado, Delgado (1997) (14), realizó un estudio para determinar la proporción de repetición de las impresiones de diagnóstico, trabajo y pasos repetidos por 16s estudiantes en la Clínica Estomatológica Central de la UPCH, encontrando un $41,1 \%$ de repeticiones. Esto ocasionó un mayor gasto en la clínica y el laboratorio dental así como un mayor tiempo utilizado para el tratamiento del paciente, afectando la satisfacción de éste. En el mismo sentido Rodríguez (1997) (13), realizó un estudio para terminar la proporción de repeticiones de actividades finales o intermedias terminadas entregadas al operador por el area de producción del 
laboratorio dental de la clínica estomatológica central de la UPCH, encontrando un 3,7\%. Estas fallas llevaron a un aumento de citas de los pacientes y mayor gasto en clínica y en el laboratorio dental.

El objetivo del presente trabajo fue identificar la frecuencia de adaptación no forzada de los colados en prótesis parcial removible y fija en pacientes de la Clínica del Adulto de la Facultad de Odontología de la Universidad Nacional Federico Villarreal.

\section{Material y métodos}

El presente trabajo, se desarrolló en el transcurso del año 2008 en la clínica odontológica de la Universidad nacional Federico Villarreal. Se evaluó la adaptación en boca de colados metálicos de las prótesis parcial removible y fija para lo cual se tomó 100 colados de prótesis tanto parcial removible como fija, provenientes del laboratorio de prótesis de la Facultad de Odontología, los cuales; fueron evaluados en el ambiente de la clínica, tanto en el aspecto de la adaptación a los pilares y piezas de tales así como la oclusión en el paciente, a través de examen clínico con iluminación artificial, equipo de examen, papel de articular, oxido de zinc, alcohol y silicona fluida. Los datos fueron recogidos con el instrumento para el recojo de datos en la cual se consignaron datos como el tipo de prótesis, si adaptó en la primera maniobra de asentamiento o si, necesitaron mas intentos con desgastes internos y luego, si se encuentra en oclusión o si también requiere desgastes y finalmente si no adaptó y requiere repetición del colado. Para ello se aplicó este instrumento a cada caso, a través del examen clínico a cada paciente, observando la adaptación de los márgenes, apoyos y aditamentos que deben estar completamente adaptados en las estructuras de las piezas dentarias en las cuales; se apoyarán en el caso de las prótesis parciales removibles, así como el sellado de las coronas, incrustaciones y perno muñones en el caso de las prótesis fijas.

\section{Resultados}

De los 100 colados que sirvieron de muestra, el 38\% (38) son prótesis parcial removible de cromo cobalto y e162\% (62) son prótesis fija de los cuales 23 perno muñones, 18 coronas individuales, 18 puentes y 3 incrustaciones.

De esta muestra, el 78\% de las prótesis parciales removibles, requirieron desgastes internos para su adaptación, ya sea de los ganchos retenedores y/u opositores como de los apoyos, el $10 \%$ se debió repetir y tan solo e1 12\% adaptó sin ningún tipo de desgate. En cuanto a las prótesis fijas, del total de perno colados, solo el $3 \%$ requirió de repetición y el resto debió hacerse algún tipo de desgaste, por otro lado de las 18 coronas, el 95\% debió recibir algún tipo de desgaste para su adaptación y un 5\% debió de repetirse, del mismo modo, en las prótesis fijas tipo puente al 65\% debieron hacerle algún tipo de desgaste interno para su adaptación, al $10 \%$ se requirió desgaste oclusal mientras que un $20 \%$ debió repetirse y tan solo un 5\% no requirió ningún tipo de desgaste. Finalmente, las incrustaciones en el $100 \%$ requirieron algún tipo de desgaste para su adaptación (Tabla 1).

\section{Discusión}

Los resultados del presente trabajo, muestran elevados porcentajes de falta de adaptación en los colados de las estructuras metálicas de los trabajos que se realizan en la Clínica Odontológica de la Facultad.

Nuestros resultados, concuerdan con los encontrados por Delgado (14) quien muestra elevados porcentajes de errores y fallas que no permitieron la adaptación de los colados tanto en coronas y puentes así como en prótesis parcial removible.

Del mismo modo, nuestros resultados son coherentes con lo encontrado por Cueto (8), Ccahuana (17) y Rodríguez (13), quienes reportaron una alta frecuencia de repeticiones de trabajos de laboratorio, producto de errores y fallas tanto en los colados que no permitieron su adaptación así como en el procesamiento de las prótesis dentales.

La eficiencia de un servicio odontológico que brinda tratamiento con prótesis dental, se mide también por la cantidad de repeticiones que se hagan en este tratamiento, vale mencionar que los errores o fallas que pueden presentar los colados $\mathrm{y}$ que no permitan su adaptación, no solo se debe al proceso del colado en el laboratorio, sino a todo el proceso de atención, que tiene que ver con este colado, es decir, al

Tabla 1. Adaptación del colado según tipo de prótesis.

\begin{tabular}{|c|c|c|c|c|}
\hline Condición & adapta & desgaste & repetir & total \\
\hline Tipo prótesis & n $(\%)$ & n $(\%)$ & n $(\%)$ & n (\%) \\
\hline Prótesis parcial removible & $5(12)$ & $30(78)$ & $3(10)$ & $30(100)$ \\
\hline Perno muñón & $0 \quad(0)$ & $22(97)$ & 1 (3) & $23(100)$ \\
\hline Coronas individúales & $0 \quad(0)$ & 17 (95) & 1 (5) & $19(100)$ \\
\hline Puente fijo & 1 (5) & $13(75)$ & $4(20)$ & 18 (100) \\
\hline Incrustaciones & $0 \quad(0)$ & $3(100)$ & $0(0)$ & $3(100)$ \\
\hline
\end{tabular}


aspecto clínico desde: el tallado pasando por las impresiones y terminando con el vaciado de estas impresiones para obtener el modelo de trabajo.

Existen principios y fundamentos que rigen el desarrollo de estos procedimientos y que se encuentran definidos y se aplican en los diseños y elaboración de estas prótesis; sin embargo ya pesar de ello, se suelen presentar algunos errores que con maniobras de desgaste se logran corregir.

Un servicio odontológico que brinde un servicio de calidad, debe tener procesos estandarizados y criterios unificados para que el resultado sea el que se espera, un servicio de calidad, con satisfacción plena del paciente.

En un servicio docente asistencial, donde los trabajos clínicos son guiados por docentes capacitados, el porcentaje de errores y fallas deberían ser mínimos; sin embargo, nuestros resultados así como de investigadores antes mencionados, nos revelan lo contrario, implicando esto malestar del paciente porque se tiene que repetir el trabajo, aumentando el número de citas e incrementando el costo, por lo que es muy importante que estos aspectos se tengan en cuenta.

Se puede concluir con nuestros resultados y los encontrados por otros investigadores que esta fase de laboratorio en la confección de las prótesis dentales, son un problema serio que afecta directamente a la calidad del servicio y por ende a la satisfacción del usuario o paciente que se tiene que resolver con el diseño de controles más estrictos en este proceso.

\section{Referencias bibliográficas}

1. Cornejo A. Calidad total. México
D.F.: McGraw Hill; 1994.

2. ¿Cómo lograr la calidad en la Clínica Dental?; 2002 [Citado febrero 2008]. Disponible en: http://www.gacetadental.com/ foyci_texto.asp?d1=marzo2002/ ciencia/\&d2=6\&d3=/marzo2002/ ciencia/6.htm.

3. Gurnik D, Twan M. Evaluating health care programs and systems. An epidemiologic perspective. Epidemiology and the delivery of health care services: methods and applications. Chicago: Rush University; 1995.

4. Rojas H. Mejoramiento de la calidad de servicios mediante la orientación al cliente y el compromiso de la empresa; 1997 [Citado febrero 2008]. Disponible en: http:// www.monografias.com/ trabajos 15/calidad-serv/ calidadserv.shtml.

5. Stoner J, Freeman E, Gilbert D. Administración. Mexico DF: Prentice Hall Hispanoamérica SA; 1996.

6. Pegorao F. Prótesis Fija. Sao Paulo: Editora Artes Médicas Ltda; 2001.

7. Shillinburg H. Fundamentos esenciales en prótesis fija. Barcelona: Quintessence S.L.; 2000.

8. Cueto J, Grillo L, Quispe L, Mosqueira J, Zelada J, Alvarado L, Villafuerte J, Uribe L. Errores más comunes en Prótesis Totales. Lima: Escuela de Tecnología Dental. Universidad Peruana Cayetano Heredia; 2002.

9. Freund V, Quintana M, Rivera E, Romero J, Sánchez M. Laboratorio Dental Dentalab SAC: informe de consultoría. Programa de Gerencia en Servicios de Salud-2002. Lima:
ESAN; 2003.

10.Domingo J. Los errores de laboratorio; 2003 [Citado febrero 2008]. Disponible en: http:// www.medicadetarragona.es/ a u l a / L a b o r a t ori o / loserroresdelaboratorio.htm.

11. Modaffore M, Soga H, Klieman C. O que devemos enviar ao laboratorio para confeccäo de uma boa prótese parcial removível. J Bras Clin Estet Odontol. 2000, 4(24):77-81.

12. Aquilino A, Taylor D. Prosthodontic laboratory and currículo survery. Part III. Fixed prosthodontic laboratory survey. J Prosthet Dent. 1984; 52:897.

13. Rodríguez C. Proporción de repeticiones de actividades finales o intermedias terminadas entregados al operador por el área de producción del Laboratorio Dental de la Clínica Estomatológica Central de la Universidad Peruana Cayetano Heredia durante el período del 12 de Nov. al 20 de Dic. de 1996 como una medida de eficiencia en Servicios Estomatológicos [Tesis para optar por el título de cirujano dentista]. [Lima]: Universidad Peruana Cayetano Heredia; 1997.

14.Delgado L. Repetición de impresiones de diagnóstico, trabajo y pasos clínicos efectuados por los alumnos de pre-grado en el curso de clínica integral del adulto de la Facultad de Estomatología en la Clínica Estomatológica Central de la Universidad Peruana Cayetano Heredia. ene. - dic. 1996 [Tesis para obtener el Título de Cirujano Dentista]. [Lima]: Universidad Peruana Cayetano Heredia; 1997.

15. Horovitz J. La calidad del servicio. Mexico D.F.: 
Interamericana SA; 1991.

16.Pérez A. Las quejas de los pacientes. Dental Economics. 1997; 3(4).

17.Ccahuana R. Número de citas por tipo de tratamiento en rehabilitación oral y endodoncia, efectuado por los alumnos del curso de clínica integral del adulto en pre-grado, en la Clínica Estomatológica Central de la Facultad de Estomatología de la
Universidad Peruana Cayetano Heredia. Enero a diciembre de 1996 [Tesis para optar por el título de cirujano dentista]. [Lima]: Universidad Peruana Cayetano Heredia; 1997. 